\title{
Adiabaticity criterion and the shortest adiabatic mode transformer in a coupled-waveguide system
}

\author{
Xiankai Sun," Hsi-Chun Liu, and Amnon Yariv \\ Department of Applied Physics and Department of Electrical Engineering, MC 128-95, \\ California Institute of Technology, Pasadena, California 91125, USA \\ *Corresponding author: xksun@caltech.edu
}

Received October 8, 2008; revised December 7, 2008; accepted December 8, 2008; posted December 12, 2008 (Doc. ID 102559); published January 26, 2009

By analyzing the propagating behavior of the supermodes in a coupled-waveguide system, we have derived a universal criterion for designing adiabatic mode transformers. The criterion relates $\varepsilon$, the fraction of power scattered into the unwanted mode, to waveguide design parameters and gives the shortest possible length of an adiabatic mode transformer, which is approximately $2 / \pi \varepsilon^{1 / 2}$ times the distance of maximal power transfer between the waveguides. The results from numerical calculations based on a transfer-matrix formalism support this theory very well. (C) 2009 Optical Society of America

OCIS codes: $060.1810,130.2790,130.3120,230.7370$.

Mode transformers have been widely used in optical communications to efficiently route and transmit light between different components. During the past two decades, they have been extensively studied in tapered fibers [1] and "spot-size converter" integrated laser diodes [2], modulators [3], optical amplifiers [4], WDM filters [5], and optical switches [6]. Today, with the development of new concepts and techniques, coupling of light between different photonic devices in hybrid material systems assumes an everincreasing importance $[7,8]$. Two schemes have been implemented to realize the mode transformationresonant coupling and adiabatic coupling. In a resonant coupler, by designing the coupling region to be of a half beat length, the light transfers from one waveguide to the other [8]. The coupler length can be made very short in this manner; however, it is practically difficult to determine the exact beat length, rendering the efficiency of power transfer uncertain and the resulting devices of dubious value. An adiabatic coupler, on the other hand, does not require a precise definition of power-transfer length $[1,9,10]$, but it has to be sufficiently long to satisfy the adiabatic condition to reduce the coupling of power into other unwanted modes. Clearly a longer coupler not only reduces the component density but also suffers from higher transmission losses and higher probability of material defects and fabrication imperfections.

The optimal design procedure of adiabatic mode transformers has been proposed in different ways. Love et al. first studied the fiber tapers, suggesting that for a given taper length the optimal delineating curve should have the local taper angle inversely proportional to the local beat length $[1,11]$. This design principle was also employed in experiments $[10,12]$. Another design concept is based on equalization of the "single-step loss" (defined as the overlap integral of the modes in two adjacent segments) along the taper $[13,14]$. Based on the stationary field distributions rather than the wave propagation behavior, those analyses did not point out the shortest taper length with which a certain coupling efficiency could be achieved. In this Letter we derive a universal cri- terion for the adiabatic mode transition in a coupledwaveguide system and suggest the shortest length of an adiabatic mode transformer for a given maximal tolerated scattering from the wanted mode into other modes during power transfer.

The mode transformer to be analyzed here is based on a coupled-waveguide system shown in Fig. 1. It consists of two waveguides, waveguide $\mathbf{1}$ and waveguide 2, placed in close proximity to each other. The refractive index or geometry of at least one waveguide is gradually varied along the propagation direction $z$. Light is coupled into this transformer at input plane $z=z_{i}(=0)$ and out at output plane $z=z_{f}$. The normalized local modes (or "supermodes"), denoted as $\mathbf{e}_{\mathbf{e}}$ for the even mode and $\mathbf{e}_{\mathbf{o}}$ for the odd mode, of this coupled-waveguide system are expressed as column vectors with their components being the amplitudes of the two individual waveguide modes [15],

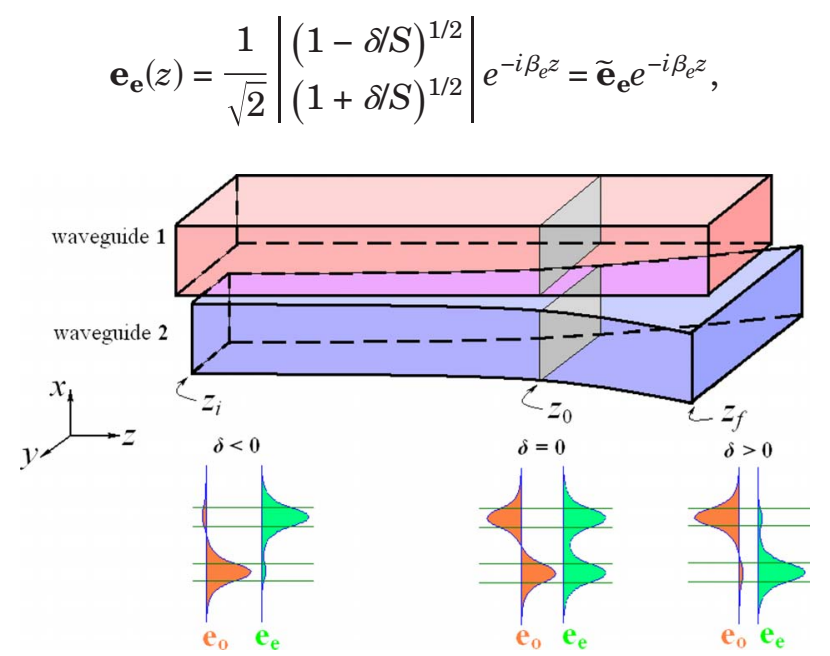

Fig. 1. (Color online) Model of the adiabatic mode transformer based on two coupled waveguides $\mathbf{1}$ and $\mathbf{2}$, placed in close proximity to each other. The geometry of waveguide 2 is gradually varied along the $z$ direction. Light is coupled in at plane $z=z_{i}(=0)$ and out at plane $z=z_{f}$. The modal profiles of the local modes $\mathbf{e}_{\mathbf{e}}$ and $\mathbf{e}_{\mathbf{o}}$ are listed at the input, phasematching, and output planes. 


$$
\mathbf{e}_{\mathbf{o}}(z)=\frac{1}{\sqrt{2}}\left|\begin{array}{c}
-(1+\delta / S)^{1 / 2} \\
(1-\delta / S)^{1 / 2}
\end{array}\right| e^{-i \beta_{o} z}=\widetilde{\mathbf{e}}_{\mathbf{o}} e^{-i \beta_{o} z},
$$

where $\delta=\left(\beta_{2}-\beta_{1}\right) / 2$ is the mismatch of propagation constants between the individual uncoupled waveguide modes, $2 S=2\left(\delta^{2}+\kappa^{2}\right)^{1 / 2}=\beta_{e}-\beta_{o}$ is the difference of propagation constants of the supermodes, and $\kappa$ is the coupling strength between waveguides $\mathbf{1}$ and $\mathbf{2}$. Note that the modal profiles Eq. (1) of the supermodes are $z$ dependent through the parameters $\delta$ and $S$. For both supermodes $\mathbf{e}_{\mathbf{e}}$ and $\mathbf{e}_{\mathbf{o}}$, when $\delta<0$ and $|\delta| \gg \kappa$, the mode substantially resides in one waveguide, and when $\delta>0$ and $\delta \gg \kappa$, the mode is substantially in the other waveguide. At the phase-matching point $z_{0}$ where $\delta=0$ the mode is evenly distributed in the two waveguides [7]. Our task is to design this coupled-waveguide system such that a supermode can transform adiabatically in the shortest distance with the parameter $\delta$ sweeping from a negative value to a positive value, so as to move its modal power spatially from one waveguide to the other while keeping the coupling to the other supermode below a certain level.

The total field in the mode transformer can be expressed as a linear combination of the supermodes with their phases accumulated $\mathbf{E}(z)=\sum_{j=e, 0} a_{j}(z) \widetilde{\mathbf{e}}_{\mathbf{j}}$ $\times \exp \left[-i \int_{0}^{z} \beta_{j}\left(z^{\prime}\right) \mathrm{d} z^{\prime}\right]$, which under paraxial approximation must satisfy the Fresnel equation $i \partial_{z} \mathbf{E}(z)$ $=\hat{B} \mathbf{E}(z)$, where $\hat{B}$ is the Fresnel operator [16]. Considering $\hat{B} \widetilde{\mathbf{e}}_{\mathbf{e}}(z)=\beta_{e}(z) \widetilde{\mathbf{e}}_{\mathbf{e}}(z)$ and $\hat{B} \widetilde{\mathbf{e}}_{\mathbf{o}}(z)=\beta_{o}(z) \widetilde{\mathbf{e}}_{\mathbf{o}}(z)$, we obtain

$$
\sum_{j=e, o}\left[\left(\partial_{z} a_{j}\right) \widetilde{\mathbf{e}}_{\mathbf{j}}+a_{j}\left(\partial_{z} \widetilde{\mathbf{e}}_{\mathbf{j}}\right)\right] \exp \left[-i \int_{0}^{z} \beta_{j}\left(z^{\prime}\right) \mathrm{d} z^{\prime}\right]=0 .
$$

Without loss of generality, if we consider the case of adiabatically transforming the mode $\mathbf{e}_{\mathbf{e}}$, then only $\mathbf{e}_{\mathbf{e}}$ is excited, and the power coupled into $\mathbf{e}_{\mathbf{o}}$ is negligible. We can take $a_{e} \approx 1$ and $a_{o} \approx 0$ so that Eq. (2) becomes $\left(\partial_{z} a_{o}\right) \widetilde{\mathbf{e}}_{\mathbf{o}} \exp [2 i \bar{S} z]+\left(\partial_{z} a_{e}\right) \widetilde{\mathbf{e}}_{\mathbf{e}}+\partial_{z} \widetilde{\mathbf{e}}_{\mathbf{e}}=0, \quad$ where $\bar{S}(z)$ $=\left[\int_{0}^{z} S\left(z^{\prime}\right) \mathrm{d} z^{\prime}\right] / z$ and $S=\left(\beta_{e}-\beta_{o}\right) / 2$. Taking the inner product with $\widetilde{\mathbf{e}}_{\mathbf{o}}^{*}$ yields $\mathrm{d} a_{o} / \mathrm{d} z=-\left(\widetilde{\mathbf{e}}_{\mathbf{o}}^{*} \cdot \partial_{z} \widetilde{\mathbf{e}}_{\mathbf{e}}\right) e^{-2 i \bar{S} z}$. It can be shown from Eq. (1) that $\widetilde{\mathbf{e}}_{\mathbf{o}}^{*} \cdot \partial_{z} \widetilde{\mathbf{e}}_{\mathbf{e}}=[2(1$ $\left.\left.+\gamma^{2}\right)\right]^{-1} \mathrm{~d} \gamma / \mathrm{d} z$ where $\gamma \equiv \delta / \kappa$. Then we have

$$
\frac{\mathrm{d} a_{o}}{\mathrm{~d} z}=\left(\frac{1}{4 i \kappa\left(1+\gamma^{2}\right)^{3 / 2}} \frac{\mathrm{d} \gamma}{\mathrm{d} z}\right) \frac{\mathrm{d}}{\mathrm{d} z}\left(e^{-2 i \bar{S} z}\right),
$$

where $\mathrm{d}(\bar{S} z) / \mathrm{d} z=S$ and $S=\kappa\left(1+\gamma^{2}\right)^{1 / 2}$ have been used. Since the factor in the first bracket on the right-hand side of Eq. (3) varies slowly compared to the exponential term $e^{-2 i \bar{S} z}$, Eq. (3) is integrated over $z$, resulting in

$$
a_{o}=-\left(\frac{1}{2 \kappa\left(1+\gamma^{2}\right)^{3 / 2}} \frac{\mathrm{d} \gamma}{\mathrm{d} z}\right) e^{-i \bar{S} z} \sin (\bar{S} z),
$$

and thus

$$
\left|a_{o}\right| \leqslant \frac{1}{2 \kappa\left(1+\gamma^{2}\right)^{3 / 2}} \frac{\mathrm{d} \gamma}{\mathrm{d} z},
$$

which imposes an upper bound on the amplitude of $a_{o}$.

In an adiabatic mode transformer, we require that the fraction of power in the unwanted mode $\mathbf{e}_{\mathbf{o}}$ be less than a certain value $\varepsilon$, or equivalently $\left|a_{o}\right|<\varepsilon^{1 / 2}$, along the propagation. Following Eq. (4), we arrive at the adiabaticity criterion in this two-mode coupledwaveguide system,

$$
\frac{1}{2 \kappa\left(1+\gamma^{2}\right)^{3 / 2}} \frac{\mathrm{d} \gamma}{\mathrm{d} z} \leqslant \varepsilon^{1 / 2} .
$$

It is straightforward that the shortest length with the largest possible $\mathrm{d} \gamma / \mathrm{d} z$ is achieved only when the equality holds. In the case that the separation between the two waveguides does not change and $\kappa$ can be regarded as a constant [17], the solution is

$$
\gamma=\frac{u}{\sqrt{1-u^{2}}}=\tan [\arcsin (u)],
$$

where $u \equiv 2 \kappa \varepsilon^{1 / 2}\left(z-z_{0}\right)$ and $z_{0}$ is the phase-matching point corresponding to a zero $\gamma$. If $\kappa$ cannot be regarded as a constant, then the factor $\kappa\left(z-z_{0}\right)$ should be replaced by $\int_{z_{0}}^{z} \kappa\left(z^{\prime}\right) \mathrm{d} z^{\prime}$. It follows from Eq. (6) that as $z-z_{0}$ varies from $-1 /\left(2 \kappa \varepsilon^{1 / 2}\right)$ to $1 /\left(2 \kappa \varepsilon^{1 / 2}\right)$, $\gamma$ covers $-\infty$ to $\infty$, which means a transformer designed with Eq. (6) can transform the mode $\mathbf{e}_{\mathbf{e}}$ from being completely in waveguide $\mathbf{1}$ at the input to completely in waveguide 2 at the output in a length $L=1 / \kappa \varepsilon^{1 / 2}$ with the fraction of power in the unwanted mode $\mathbf{e}_{\mathbf{o}}$ below $\varepsilon$. In other words, with this optimal design, $\varepsilon$ has the least value $(1 / \kappa L)^{2}$, which is in agreement with previous studies indicating the scattered power being proportional to $L^{-2}$ [9]. Considering that a resonant coupler requires the least distance $\pi / 2 \kappa$ for maximal power transfer from one waveguide to the other, it takes $2 / \pi \varepsilon^{1 / 2}$ times this distance to achieve the mode transformation in an adiabatic coupler.

To verify the above theory, we employ a transfermatrix formalism to investigate the coupling efficiency of adiabatic mode transformers with different distributions of $\gamma$ along $z$. For the coupled-waveguide system shown in Fig. 1, the amplitudes of each waveguide mode, $b_{1}(z)$ and $b_{2}(z)$, satisfy the coupled-mode equations

$$
\frac{\mathrm{d}}{\mathrm{d} z}\left[\begin{array}{l}
b_{1} \\
b_{2}
\end{array}\right]=\left[\begin{array}{cc}
-i \beta_{1} & -i \kappa \\
-i \kappa & -i \beta_{2}
\end{array}\right]\left[\begin{array}{l}
b_{1} \\
b_{2}
\end{array}\right] \equiv \mathbf{C}\left[\begin{array}{l}
b_{1} \\
b_{2}
\end{array}\right],
$$

which leads to the transfer relation for small $\Delta z$ :

$$
\left[\begin{array}{l}
b_{1}(z+\Delta z) \\
b_{2}(z+\Delta z)
\end{array}\right]=\exp (\mathbf{C}(z) \cdot \Delta z)\left[\begin{array}{l}
b_{1}(z) \\
b_{2}(z)
\end{array}\right],
$$

where 


$$
\mathbf{C}(z)=-i \bar{\beta}(z) \mathbf{I}+i \kappa\left[\begin{array}{cc}
\gamma(z) & -1 \\
-1 & -\gamma(z)
\end{array}\right],
$$

with $\mathbf{I}$ being the identity matrix and $\bar{\beta}=\left(\beta_{1}+\beta_{2}\right) / 2$. Only the second term in the expression of $\mathbf{C}(z)$ is important, since the first term just provides an overall phase factor for both $b_{1}$ and $b_{2}$. Light is launched into the transformer at the input plane $z_{i}$ with the mode substantially resident in waveguide 1 so that $b_{1}\left(z_{i}\right)$ $=1$ and $b_{2}\left(z_{i}\right)=0$. The coupling efficiency of the mode transformer is defined as $\left|b_{2}\left(z_{f}\right)\right|^{2}$, the fraction of power in waveguide 2 at the output plane $z_{f}$. Let us take $\kappa$ to be $40 \mathrm{~mm}^{-1}$, which was calculated from a Si/ III-V supermode laser structure in [17], and assume a practical $\gamma$ to vary from -10 to 10 across the mode transformer. Figure 2 plots the coupling efficiency as a function of the transformer length $L\left(=z_{f}-z_{i}\right)$ for devices designed with $\gamma$ distributed according to Eq. (6), and to some common odd functions such as $u, u^{3}$, and $\sin (u)$. For the optimal distribution $\tan [\arcsin (u)]$, a transformer with length $L=200 \mu \mathrm{m}$ is sufficiently adiabatic to transfer the power from waveguide 1 to waveguide 2 . With this length the fraction of power scattered from $\mathbf{e}_{\mathbf{e}}$ to $\mathbf{e}_{\mathbf{o}}$ is $\left(\frac{1}{8}\right)^{2}$ based on $\varepsilon=(1 / \kappa L)^{2}$. Devices with other $\gamma$ distributions require a substantially longer length to achieve the same $\varepsilon$ level.

The fundamental reason why an adiabatic mode transformer favors the $\tan [\arcsin (u)]$-shaped $\gamma$ distribution is that only by following this distribution can the power scattered into the unwanted mode have the smallest least upper bound. In this numerical case, since the $\gamma$ value of -10 leads to $\left|a_{o}\right| \approx \frac{1}{20}$ at the input plane $z_{i}$ and the fraction of power coupled from $\mathbf{e}_{\mathbf{e}}$ to $\mathbf{e}_{\mathbf{o}}$ is $\left(\frac{1}{8}\right)^{2}$ during the transformation, it is expected that at the output plane $z_{f}$ the maximal fraction of power in the mode $\mathbf{e}_{\mathbf{o}}$ satisfies $\left(\frac{1}{8}-\frac{1}{20}\right)^{2}$ $\leqslant\left|a_{o}\right|_{\max }^{2} \leqslant\left(\frac{1}{8}+\frac{1}{20}\right)^{2}$, the specific value depending on the propagation phase in the transformer. Figure 3 displays the fraction of power in modes $\mathbf{e}_{\mathbf{e}}$ and $\mathbf{e}_{\mathbf{o}}$, calculated from the transfer-matrix method, in a

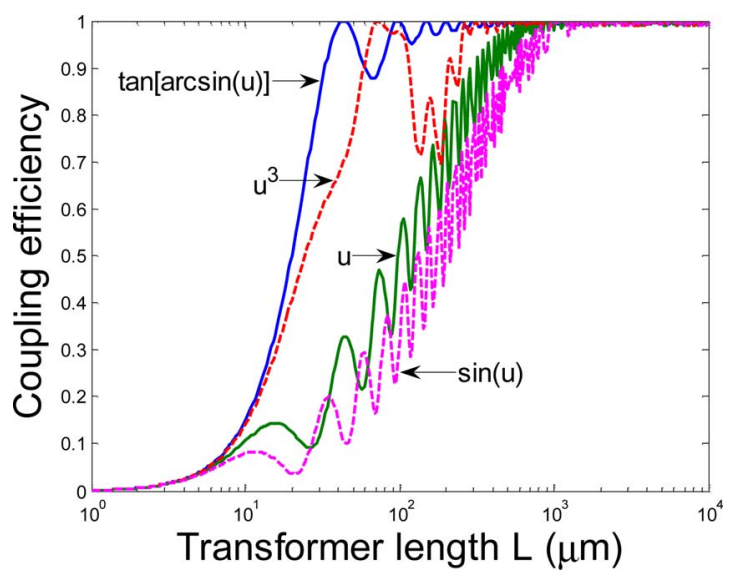

Fig. 2. (Color online) Coupling efficiency as a function of the transformer length $L\left(=z_{f}-z_{i}\right)$ for devices designed with $\gamma$ distributions of $\tan [\arcsin (u)], u, u^{3}$, and $\sin (u)$. The optimal design with $\gamma$ distributed with $\tan [\arcsin (u)]$ has the smallest length to achieve the same level of adiabatic mode transformation.

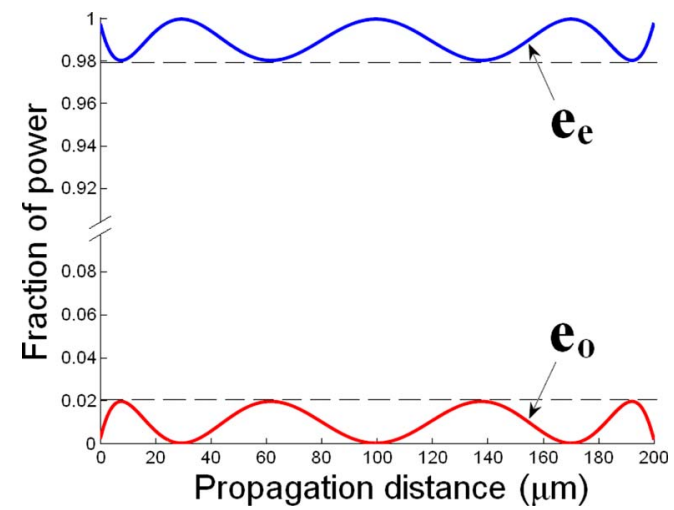

Fig. 3. (Color online) Fraction of power in modes $\mathbf{e}_{\mathbf{e}}$ and $\mathbf{e}_{\mathbf{o}}$ along the $+z$ direction in a 200 - $\mu$ m-long mode transformer with the optimal design. The equiripple behavior for both modes indicates the minimal power scattering.

200- $\mu$ m-long mode transformer with the optimal shape. The equiripple behavior for both modes is evident, and the fraction of power in $\mathbf{e}_{\mathbf{o}}$ is bounded by 0.02 , which falls in the expected range.

This work was supported by Defense Advanced Research Projects Agency (DARPA) MTO office (J. Shah) under contract N66001-07-1-2058.

\section{References}

1. J. D. Love, W. M. Henry, W. J. Stewart, R. J. Black, S. Lacroix, and F. Gonthier, IEE Proc.-J: Optoelectron. 138, 343 (1991).

2. K. Kasaya, Y. Kondo, M. Okamoto, O. Mitomi, and M. Naganuma, Electron. Lett. 29, 2067 (1993).

3. N. Yoshimoto, K. Kawano, Y. Hasumi, H. Takeuchi, S. Kondo, and Y. Noguchi, IEEE Photon. Technol. Lett. 6, 208 (1994).

4. D. Sigogne, A. Ougazzaden, D. Meichenin, B. Mersali, A. Carenco, J. C. Simon, I. Valiente, C. Vassallo, and L. Billes, Electron. Lett. 32, 1403 (1996).

5. A. S. Kewitsch, G. A. Rakuljic, P. A. Willems, and A. Yariv, Opt. Lett. 23, 106 (1998).

6. K. Kawano, M. Kohtoku, N. Yoshimoto, S. Sekine, and Y. Noguchi, Electron. Lett. 30, 353 (1994).

7. A. Yariv and X. K. Sun, Opt. Express 15, 9147 (2007).

8. M. Galarza, D. Van Thourhout, R. Baets, and M. Lopez-Amo, Opt. Express 16, 8350 (2008).

9. S. G. Johnson, P. Bienstman, M. A. Skorobogatiy, M. Ibanescu, E. Lidorikis, and J. D. Joannopoulos, Phys. Rev. E 66, 066608 (2002).

10. F. Xia, V. M. Menon, and S. R. Forrest, IEEE J. Sel. Top. Quantum Electron. 11, 17 (2005).

11. J. D. Love and W. M. Henry, Electron. Lett. 22, 912 (1986).

12. T. Bakke, C. T. Sullivan, and S. D. Mukherjee, J. Lightwave Technol. 20, 1188 (2002).

13. B. Hubner, G. Vollrath, R. Ries, C. Greus, H. Janning, E. Ronneberg, E. Kuphal, B. Kempf, R. Gobel, F. Fiedler, R. Zengerle, and H. Burkhard, IEEE J. Sel. Top. Quantum Electron. 3, 1372 (1997).

14. S. R. Park and B. H. O, IEEE Photon. Technol. Lett. 13, 675 (2001).

15. A. Yariv, IEEE J. Quantum Electron. 9, 919 (1973).

16. G. Lifante, Integrated Photonics: Fundamentals (Wiley, 2003).

17. X. K. Sun and A. Yariv, J. Opt. Soc. Am. B 25, 923 (2008). 